\title{
Epiphytic angiosperms in a mountain forest in southern Bahia, Brazil
}

\author{
Paula Leitman ${ }^{1,5}$, André Amorim ${ }^{2,3}$, Luiz Menini Neto ${ }^{4}$ \& Rafaela C. Forzza ${ }^{1}$ \\ ${ }^{1}$ Jardim Botânico do Rio de Janeiro, Rua Pacheco Leão 915, Jardim Botânico, Rio de Janeiro, RJ, Brazil, \\ CEP: 22460-030 \\ ${ }^{2}$ Universidade Estadual de Santa Cruz, Departamento de Ciências Biológicas, Salobrinho, Ilhéus, BA, \\ Brazil, CEP: 45662900 \\ ${ }^{3}$ Centro de Pesquisas do Cacau - Herbário CEPEC, Caixa Postal 07, Itabuna, Bahia, Brazil, CEP: \\ 45660970 \\ ${ }^{4}$ Centro de Ensino Superior de Juiz de Fora, Campus Arnaldo Janssen - Rua Luz Interior 345, Santa Luzia, \\ Juiz de Fora, MG, Brazil, CEP: 36030-776 \\ ${ }^{5}$ Corresponding author: Paula Leitman,e-mail: paulaleitman@gmail.com
}

LEITMAN, P., AMORIM, A., MENINI NETO, L., FORZZA, R.C. Epiphytic angiosperms in a mountain forest in southern Bahia, Brazil. Biota Neotropica. 14(2): e20130010. http://dx.doi.org/10.1590/ S1676-06032014001013

\begin{abstract}
The Atlantic Forest in southern Bahia State, Brazil, has high levels of diversity and vascular plant endemism. There have been few floristic surveys of the mountain forests there, however, especially those focusing on herbs and canopy plants, with studies of Atlantic Forest epiphytes having been limited to the southern and southeastern region of that country. The present study therefore surveyed the epiphytic angiosperms in the Serra das Lontras National Park (SLNP), the distribution of their species among genera and families, and their biogeographical patterns. Fieldwork was performed between February 2011 and March 2012. Collections were made along roads and trails and phorophytes were occasionally climbed and fallen trees and branches examined in order to sample canopy species. The study site demonstrated high epiphyte richness (256 spp.), one of the highest recorded in the Atlantic Forest. The richest families are Orchidaceae, Bromeliaceae, and Araceae, reaffirming the patterns reported in previous major reviews on epiphytes. The species exhibit high degrees of endemism $(45 \%)$ to the Atlantic Forest, especially among the Bromeliaceae and Orchidaceae. One new occurrence to Brazil, four to the Atlantic Forest and 30 to Bahia state are registered. The SLNP shares more species with areas of southern and southeastern Brazil than with other northeastern states.
\end{abstract}

Keywords: Atlantic forest, Araceae, Bromeliaceae, Orchidaceae, biogeographical patterns.

LEITMAN, P., AMORIM, A., MENINI NETO, L., FORZZA, R.C. Angiospermas epífitas de uma floresta montana no sul da Bahia, Brasil. Biota Neotropica. 14(2): e20130010. http://dx.doi.org/10.1590/ S1676-06032014001013

Resumo: A Floresta Atlântica do sul da Bahia apresenta elevados níveis de diversidade e endemismo de plantas vasculares. No entanto, poucos levantamentos florísticos foram realizados em áreas de floresta montana, especialmente para ervas e plantas de dossel. Estudos com epífitas estão concentrados principalmente em áreas do Sul e Sudeste do Brasil. Desta forma, o presente estudo realizou o levantamento das angiospermas epífitas do Parque Nacional da Serra das Lontras (PNSL) e verificou a distribuição das espécies pelas famílias e gêneros e seus padrões biogeográficos. As coletas foram realizadas entre fevereiro de 2011 e março de 2012 nas trilhas e estradas de acesso. Alguns forófitos foram escalados e árvores e ramos caídos foram examinados com o intuito de coletar espécies restritas ao dossel. A área de estudo apresentou alta diversidade de epífitas (256 spp.), uma das maiores já registradas para a Floresta Atlântica. As famílias com maior riqueza são Orchidaceae, Bromeliaceae e Araceae, reafirmando os padrões encontrados anteriormente em revisões do tema. As espécies apresentam alto grau de endemismo (45\%) à Floresta Atlântica, especialmente em Bromeliaceae e Orchidaceae. São registradas uma nova ocorrência para o Brasil, quatro para a Floresta Atlântica e 30 para a Bahia. O PNSL possui mais espécies em comum com o Sul e o Sudeste do que com a região Nordeste.

Palavras-chave: Floresta Atlântica, Araceae, Bromeliaceae, Orchidaceae, padrões biogeográficos. 


\section{Introduction}

Epiphytes spend most or all of their life cycles growing on other plants (phorophytes), taking advantage of their mechanical support (Benzing 1990). Epiphytes account for approximately $10 \%$ of world's vascular flora and are found in 84 angiosperm families (Gentry \& Dodson 1987, Kersten 2010). Araceae, Bromeliaceae, and Orchidaceae are the most successful epiphytic angiosperm families, and the latter is responsible for over $70 \%$ of total richness.

Bromeliaceae is a typically American family, while the largest genera of Araceae and Orchidaceae (e.g., Anthurium, Epidendrum, Lepanthes, Philodendron and Pleurothallis s.l.) are likewise endemic to that continent. The Neotropical region therefore hosts the greater part of world's epiphytic flora ( $>15.500$ spp.), which are mainly concentrated in Central America, the Andes, northwestern Amazonia and the Atlantic Forest (Gentry \& Dodson 1987, Nieder et al. 2001). Of the 14.500 vascular plants known to inhabit the Atlantic Forest, 3.000-4.000 are epiphytes (Stehmann et al. 2009, Kersten 2010). The Atlantic Forest has large latitudinal (from $3^{\circ} \mathrm{S}$ to $30^{\circ} \mathrm{S}$ ) and altitudinal (from sea level up to $2.890 \mathrm{~m}$ ) extensions and significant rainfall variations - resulting in high habitat heterogeneity and an extraordinary diversity of organisms (Oliveira-Filho \& Fontes 2000).

Southern Bahia is considered an area of great biological importance, with elevated levels of richness and endemism in its arboreal component (Mori et al. 1983, Thomas et al. 1998, Martini et al. 2007, Thomas et al. 2009, Rocha \& Amorim 2012), although surveys of humid montane forests have been scarce, especially those focusing on terrestrial and epiphytic herbs (Amorim et al. 2009, Matos et al. 2010). The recently created Serra das Lontras National Park comprises a vast remnant of montane Atlantic Forest in southern Bahia (Save Brasil et al. 2009).

A preliminary survey in the park, covering only a small area, recorded 709 angiosperm species, of which $158(22.3 \%)$ were epiphytes (Amorim et al. 2009). These authors highlighted the fact that many of the species encountered, including many epiphytes (e.g., Fuchsia regia [Vell.] Munz, Nematanthus lanceolatus [Poir.] Chautems, and Vriesea longicaulis Mez), were previously known only from mountainous areas in southern and southeastern Brazil.

For the preliminary survey there was no effort focused on this habit, which presents peculiarities that hinder their sampling, including the height they occur on the phorophyte and the small sizes of some groups (e.g., Peperomia and Pleurothallis s.l.). We therefore sought to inventory the composition of epiphytic angiosperms in the Serra das Lontras National Park to investigate their distributions into genera and families and their biogeographical patterns to increase our knowledge of this group in the park and in northeastern Brazil.

\section{Material and methods}

Serra das Lontras National Park (SLNP) is located in southern Bahia State (Figure 1), Brazil, in the municipalities of Arataca, São José da Vitória, and Una $\left(15^{\circ} 07^{\prime}-15^{\circ} 15^{\prime} \mathrm{S}\right.$ and $\left.39^{\circ} 15^{\prime}-39^{\circ} 25^{\prime} \mathrm{W}\right)$. The park comprises approximately 11.000 hectares at altitudes that vary from 400 to $1.000 \mathrm{~m}$. Located $30 \mathrm{~km}$ from the coast, the mountains act as a first barrier to humid air masses moving inland from the ocean, resulting in mean annual precipitation rates of $1.300-1.600 \mathrm{~mm}$, without a marked dry season, and a mean annual temperature of $24^{\circ} \mathrm{C}$ (classified as Af in the Koppen system) (Peel et al. 2007, Amorim et al. 2009, Nacif et al. 2009).

Situated in the Atlantic Forest domain, the SLNP comprises a vegetation mosaic of anthropogenic areas mixed with wellpreserved submontane forests (with canopies $>30 \mathrm{~m}$ ) at lower altitudes and cloud forests above $800 \mathrm{~m}$ with smaller trees with twisted trunks bearing bryophytes (Stadtmüller 1987).

A total floristic inventory of the SLNP has been underway for the last eight years, and preliminary results have already been published (Amorim et al. 2009; Save Brasil et al. 2009). Visits to the area were made every two months, from February/ 2011 to March/2012 to survey the epiphytic angiosperms. Collections were made along roads and trails in order to cover the largest possible areas, and some living phorophytes were occasionally climbed and fallen trees examined to collect canopy species. Accidental epiphytes were not considered in this survey. Informations on the type of epiphytism (characteristic holoepiphyte, facultative holoepiphyte, hemiepiphyte) were obtained from local observations and complemented with informations from literature. This was done in order to have more accurate data, since many facultative species might have been seen only as epiphytes in the area or might occur as terrestrial or rupiculous in parts of the park that were not visited. All fertile material was deposited at the RB and CEPEC herbaria, while sterile specimens were incorporated into the exsitu collection at the Rio de Janeiro Botanical Garden.

Specimens collected during the general floristic inventory, as well as those from the present survey, were identified to the species level, when possible, with the help of specialists and specific literature (e.g., the Flora Neotropica). Nomenclature and species concepts follow the List of Species of the Brazilian Flora - LSBF (2013). Distribution and endemism data were obtained from the LSBF for taxa identified to the species level.

A Rapid Color Guide, published by The Field Museum, was prepared and images on most species can be seen at www. fm2.fieldmuseum.org/plantguides/guideimages.asp?ID=545.

\section{Results}

The Serra das Lontras National Park shows high epiphyte richness, with a total of 256 species identified, representing 87 genera and 18 families (Table 1) - making it one of the richest sites for epiphytic angiosperms in the Atlantic Forest. Of the published checklists, only Macaé de Cima in Rio de Janeiro State reported more epiphyte species (275 spp.) (Fontoura et al. 1997).

The most important families encountered were Orchidaceae (106 spp.), Bromeliaceae (64 spp.), Araceae (31 spp.), and Piperaceae (14 spp.). Together, these families encompassed more than $80 \%$ of the epiphyte flora at SLNP. The species distributions within the families were very unequal, however, with 11 families being represented by less than five species.

The richest genera were Vriesea (22 spp.), Peperomia and Stelis (14 spp. each), Anthurium (13 spp.), Aechmea and Philodendron (12 spp. each), Epidendrum (11 spp.), and Hohenbergia (10 spp.). The species distributions within the genera were very unequal, with half of them (43 genera) being represented by only a single species, and $82 \%$ by less than five species.

With many ornamental species, 10 epiphytes registered for the SLNP are cited on the Red Book of Brazilian Flora 
Epiphytic angiosperms in a mountain forest in southern Bahia, Brazil

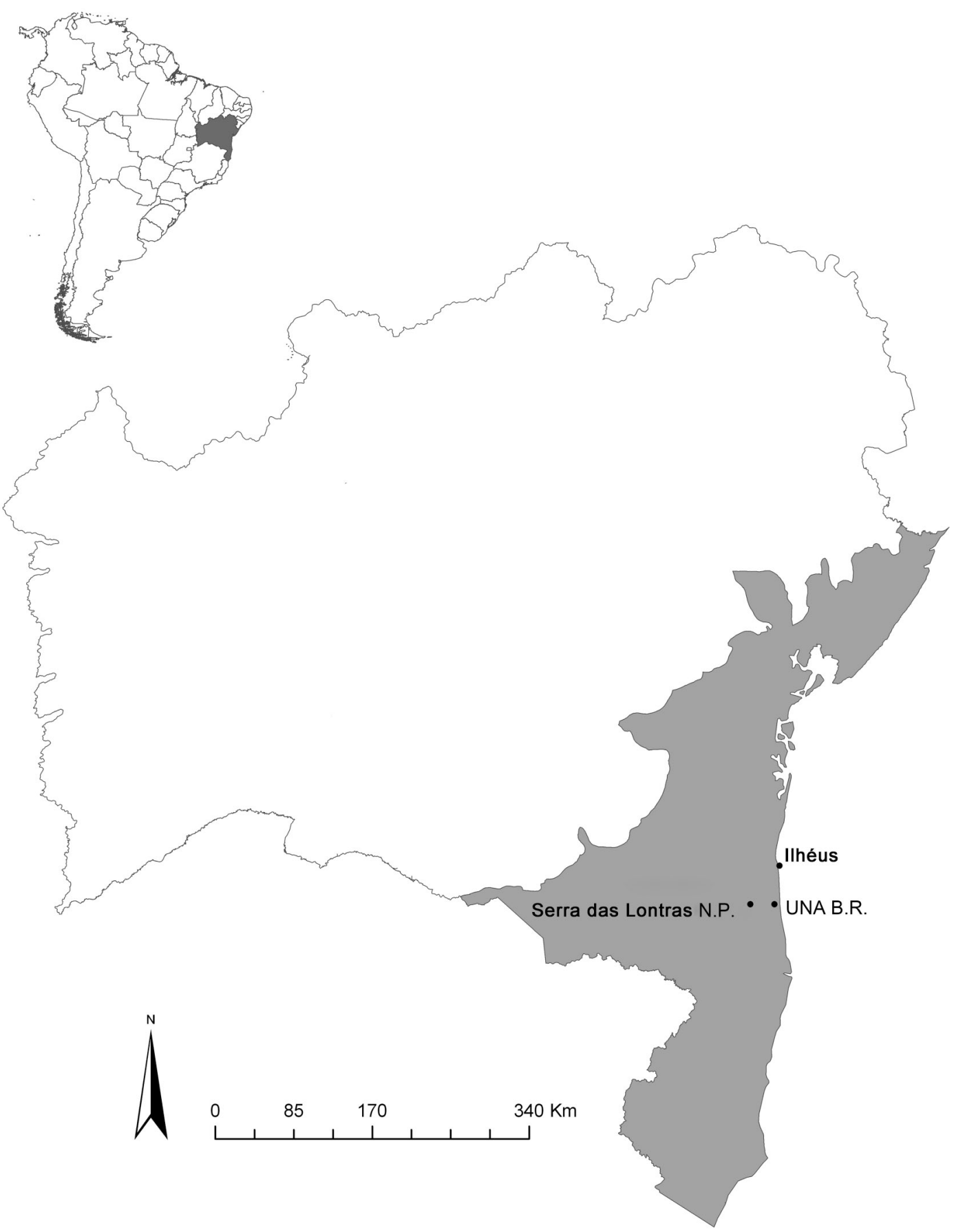

Figure 1. Map showing the location of the studied area (Serra das Lontras National Park); in gray is the Atlantic Forest limits. N.P. - National Park; B.R. - Biological Reserve/Mapa de localização da área de estudo (Parque Nacional da Serra das Lontras); em cinza está demarcado o limite da Floresta Atlântica.

(Martinelli \& Moraes 2013). Of this total, three are "Critically endangered", five are "Endangered", and two are "Vulnerable". Four are considered to be "Data Deficient" (Table 1).

\section{Discussion}

The high numbers of species observed in Araceae, Bromeliaceae, Orchidaceae, and Piperaceae were consonant with global patterns of epiphytic distribution among angiosperm families, as reported by Madison (1977) and Gentry \& Dodson (1987). Similar results were reported for the
Neotropical region (e.g., Sudgen \& Robins 1979, Gentry \& Dodson 1987, Wolf \& Flamenco-S 2003, Küper et al. 2004) and the tropical and subtropical Atlantic Forest (e.g., Fontoura et al. 1997, Borgo \& Silva 2003, Giongo \& Waechter 2004, Kersten \& Silva 2001, Buzatto et al. 2008, Martins et al. 2008, Menini Neto et al. 2009, Mania \& Monteiro 2010).

Table 2 compares the SLNP and other Atlantic Forest areas with high epiphyte richness. The elevated numbers of species of Bromeliaceae, Araceae, and Gesneriaceae is notable, representing the highest epiphyte richness ever recorded for these families in the Atlantic Forest. Vriesea, Stelis, Anthurium, 
Table 1. Epiphytic Angiosperms registered for Serra das Lontras National Park, Bahia, Brazil. ${ }^{1}{ }^{*}$ New occurence to Bahia state; ** New occurence to the Atlantic Forest; *** New occurence to Brazil. ${ }^{2}$ AA - André M. Amorim; AF - André P. Fontana; AJ - Alessandra B. Jardim; JJ - Jomar G. Jardim; JP - José Lima da Paixão; ML - Márdel M. Lopes; PF - Pedro Fiaschi; PL - Paula Leitman; RB - Rafael X. Borges; RP - Ricardo O. Perdiz; WT - Wm. Wayt Thomas. ${ }^{3}$ Epi - Characteristic Holoepiphyte; Fac - Facultative Holoepiphyte; Hep - Hemiepiphyte. ${ }^{4}$ CAF - CerradoAtlantic Forest; Di - Disjunct distribution Amazon-Atlantic Forest; En - Endemic to Atlantic Forest; WD - Wide distribution; O - Other; "-." - not applicable. ${ }^{5} \mathrm{CR}$ - Criticaly endangered; DD - Data deficient; EN - Endangered; VU - Vulnerable.

\begin{tabular}{|c|c|c|c|c|c|}
\hline Family & Specie $^{1}$ & Voucher $^{2}$ & Habit $^{3}$ & Distribution $^{4}$ & $\begin{array}{c}\text { Threat } \\
\text { category }^{5}\end{array}$ \\
\hline Acanthaceae & Clistax bahiensis Profice \& Leitman & AA 5301 & Hep & En & \\
\hline \multirow[t]{31}{*}{ Araceae } & Anthurium boudetii Nadruz * & AA 6399 & Fac & En & \\
\hline & Anthurium gladiifolium Schott & AA 5770 & Epi & En & \\
\hline & Anthurium gracile (Rudge) Lindl. ** & PL 211 & Epi & WD & \\
\hline & Anthurium intermedium Kunth & PL 165 & Fac & En & \\
\hline & Anthurium jilekii Schott & PL 547 & Hep & $\mathrm{O}$ & \\
\hline & Anthurium pentaphyllum (Aubl.) G.Don & $\begin{array}{c}\text { AA } \\
5770 \mathrm{a}\end{array}$ & Hep & WD & \\
\hline & Anthurium scandens (Aubl.) Engl. & ML 1473 & Hep & WD & \\
\hline & Anthurium solitarium Schott & AA 6610 & Fac & WD & \\
\hline & Anthurium sp. 1 & PL 417 & Epi & - & \\
\hline & Anthurium sp. 2 & JJ 4919 & Epi & - & \\
\hline & Anthurium sp. 3 & PL 324 & Epi & - & \\
\hline & Anthurium sp. 4 & JJ 4389 & Epi & - & \\
\hline & Anthurium sp. 5 & JJ 4337 & Epi & - & \\
\hline & Heteropsis oblongifolia H.B.K. & JJ 4886 & Hep & WD & \\
\hline & Monstera adansonii Schott & JP 956 & Hep & WD & \\
\hline & Philodendron cordatum Kunth & AA 6580 & Hep & En & \\
\hline & Philodendron edmundoi G.M.Barroso * & JJ 4898 & Hep & En & \\
\hline & Philodendron fragrantissimum (Hook.) G.Don & ML 1449 & Hep & WD & \\
\hline & Philodendron hederaceum (Jacq.) Schott & AA 5960 & Нер & WD & \\
\hline & Philodendron insigne Schott & JJ 4896 & Fac & $\mathrm{O}$ & \\
\hline & Philodendron ornatum Schott & AA 6727 & Hep & WD & \\
\hline & Philodendron pedatum (Hook.) H.B.K. & AA 6599 & Hep & WD & \\
\hline & Philodendron propinquum Schott & PL 202 & Hep & En & \\
\hline & Philodendron recurvifolium Schott & PL 287 & Hep & En & \\
\hline & Philodendron rudgeanum Schott & AA 5296 & Hep & $\mathrm{Di}$ & \\
\hline & Philodendron surinamense (Miq.) Engl. & PL 119 & Hep & Di & \\
\hline & Philodendron aff. williamsii Hook. f. & PL 561 & Hep & - & \\
\hline & Rhodospatha latifolia Poepp. & JJ 4842 & Hep & WD & \\
\hline & Rhodospatha oblongata Poepp. & PL 428 & Hep & Di & \\
\hline & Stenospermation spruceanum Schott & AA 5298 & Epi & $\mathrm{Di}$ & \\
\hline & Syngonium vellozianum Schott & $\begin{array}{c}\text { AA } \\
5960 \mathrm{a}\end{array}$ & Hep & WD & \\
\hline Araliaceae & Oreopanax capitatus (Jacq.) Decne. \& Planch. & AA 6568 & Hep & WD & \\
\hline \multirow[t]{5}{*}{ Begoniaceae } & Begonia convolvulacea A.DC. & AA 5280 & Hep & En & \\
\hline & Begonia fruticosa (Klotzsch) A.DC. & AA 5265 & Hep & En & \\
\hline & Begonia aff. itaguassuensis Brade & PL 338 & Hep & - & \\
\hline & Begonia radicans Vell. & AA 5275 & Hep & En & \\
\hline & Begonia grisea A.DC. ** & PL 424 & Fac & $\mathrm{O}$ & \\
\hline \multirow[t]{12}{*}{ Bromeliaceae } & Aechmea burlemarxii E. Pereira & PL 378 & Fac & En & \\
\hline & Aechmea conifera L.B.Sm. ${ }^{*}$ & PL 309 & Fac & En & \\
\hline & Aechmea froesii (L.B.Sm.) Leme \& J.A.Siqueira & AA 6594 & Fac & En & \\
\hline & Aechmea gustavoi J.A.Siqueira \& Leme & PL 262 & $\mathrm{Fac}$ & En & CR \\
\hline & Aechmea multiflora L.B.Sm. & PL 395 & Fac & En & \\
\hline & Aechmea nudicaulis Griseb. & AA 7217 & Fac & $\mathrm{CAF}$ & \\
\hline & Aechmea patentissima (Mart. ex Schult. \& Schult. f.) Baker & PL 129 & Fac & Di & \\
\hline & Aechmea subintegerrima (Philcox) Leme & ML 1484 & Epi & En & \\
\hline & Aechmea tentaculifera Leme et al. & ML 1491 & Epi & En & \\
\hline & Aechmea turbinocalyx $\mathrm{Mez}$ & PL 141 & Epi & En & \\
\hline & Aechmea viridostigma Leme \& H.Luther & JJ 4680 & Epi & En & \\
\hline & Aechmea sp. & JJ 5431 & Epi & - & \\
\hline
\end{tabular}


Table 1. Continued.

\begin{tabular}{|c|c|c|c|c|c|}
\hline Family & Specie $^{1}$ & Voucher $^{2}$ & Habit $^{3}$ & Distribution $^{4}$ & $\begin{array}{c}\text { Threat } \\
\text { category }^{5}\end{array}$ \\
\hline & Billbergia euphemiae E.Morren & AA 5752 & Fac & En & \\
\hline & Billbergia saundersii Bull & JP 976 & Epi & En & \\
\hline & Canistrum camacaense Martinelli \& Leme & AA 5751 & Fac & En & EN \\
\hline & Canistrum montanum Leme & AA 5269 & Epi & En & $\mathrm{EN}$ \\
\hline & Guzmania lingulata (L.) Mez & RB 591 & Fac & WD & \\
\hline & Hohenbergia augusta (Vell.) E.Morren * & PL 149 & Fac & En & \\
\hline & Hohenbergia brachycephala L.B.Sm. & PL 439 & Epi & En & \\
\hline & Hohenbergia capitata Schult. \& Schult.f. & AA 6602 & Epi & En & \\
\hline & Hohenbergia edmundoi L.B.Sm. \& Read & JJ 4920 & Epi & En & \\
\hline & Hohenbergia minor L.B.Sm. & PL 158 & Epi & En & \\
\hline & Hohenbergia sp. 1 & PL 398 & Epi & - & \\
\hline & Hohenbergia sp. 2 & PL 134 & Epi & - & \\
\hline & Hohenbergia sp. 3 & PL 307 & Epi & - & \\
\hline & Hohenbergia sp. 4 & PL 391 & Epi & - & \\
\hline & Hohenbergia sp. 5 & PL 305 & Epi & - & \\
\hline & Lymania azurea Leme & JJ 5316 & Epi & En & EN \\
\hline & Neoregelia azevedoi Leme & AA 6624 & Epi & En & \\
\hline & Neoregelia crispata Leme & AA 5991 & Epi & En & \\
\hline & Neoregelia kerryi Leme & AA 5807 & Fac & En & \\
\hline & Neoregelia pauciflora L.B.Sm. & AA 6590 & Epi & En & \\
\hline & Neoregelia wilsoniana M.B.Foster & PL 267 & Epi & En & \\
\hline & Nidularium innocentii Lem. & AA 6696 & Fac & En & \\
\hline & Nidularium procerum Lindm. & $\begin{array}{c}\text { WT } \\
14093 b\end{array}$ & Fac & En & \\
\hline & Portea filifera L.B.Sm. & PL 435 & Epi & En & \\
\hline & Portea nana Leme & AA 6597 & Epi & En & $\mathrm{EN}$ \\
\hline & Quesnelia clavata Amorim \& Leme & AA 5351 & Fac & En & \\
\hline & Racinaea spiculosa (Griseb.) M.A.Spencer \& L.B.Sm. & AA 5820 & Epi & WD & \\
\hline & Tillandsia geminiflora Brong. & AA 6391 & Epi & CAF & \\
\hline & Tillandsia sprengeliana Klotzsch ex Mez & JJ 4895 & Epi & $\mathrm{CAF}$ & \\
\hline & Tillandsia stricta Sol. & AA 6086 & Epi & $\mathrm{CAF}$ & \\
\hline & Vriesea breviscapa (E.Pereira \& I.A.Penna) Leme & PL 208 & Epi & En & DD \\
\hline & Vriesea dictyographa Leme & AA 5303 & Epi & En & \\
\hline & Vriesea drepanocarpa (Baker) Mez & PL 380 & Epi & En & \\
\hline & Vriesea duvaliana E.Morren & JP 965 & Epi & En & \\
\hline & Vriesea ensiformis (Vell.) Beer & JP 975 & Epi & En & \\
\hline & Vriesea flammea L.B.Sm. & RB 590 & Epi & En & \\
\hline & Vriesea guttata Linden \& André * & PL 489 & Epi & En & \\
\hline & Vriesea longiscapa Ule & AA 5797 & Epi & En & \\
\hline & Vriesea longisepala A.F.Costa & AA 6758 & Epi & En & \\
\hline & Vriesea paratiensis E.Pereira & AA 6646 & Epi & Di & \\
\hline & Vriesea procera (Mart. ex Schult. \& Schult.f.) Wittm. & PL 368 & Epi & $\mathrm{O}$ & \\
\hline & Vriesea regnelli $\mathrm{Mez}$ & AA 6759 & Epi & En & \\
\hline & Vriesea rhodostachys L.B.Sm. & PL 301 & Epi & En & DD \\
\hline & Vriesea rodigasiana E.Morren & PL 205 & Epi & En & \\
\hline & Vriesea ruschii L.B.Sm. & PL 150 & Epi & En & \\
\hline & Vriesea simplex (Vell.) Beer & PL 345 & Epi & $\mathrm{O}$ & \\
\hline & Vriesea tijucana E. Pereira & AA 6097 & Epi & En & \\
\hline & Vriesea vagans (L.B.Sm.) L.B.Sm. * & AA 6688 & Epi & En & \\
\hline & Vriesea sp. 1 & PL 289 & Epi & - & \\
\hline & Vriesea sp. 2 & PL 114 & Epi & - & \\
\hline & Vriesea sp. 3 & PL 181 & Epi & - & \\
\hline & Vriesea $\mathrm{sp} .4$ & PL 442 & Epi & - & \\
\hline \multirow[t]{3}{*}{ Cactaceae } & Epiphyllum phyllanthus (L.) Haw. & JJ 4401 & Epi & WD & \\
\hline & Lepismium cruciforme (Vell.) Miq. & PL 230 & Epi & $\mathrm{O}$ & \\
\hline & Rhipsalis floccosa Salm-Dyck ex Pfeiff. & PL 200 & Epi & En & \\
\hline
\end{tabular}


Table 1. Continued.

\begin{tabular}{|c|c|c|c|c|c|}
\hline Family & Specie $^{1}$ & Voucher $^{2}$ & Habit $^{3}$ & Distribution $^{4}$ & $\begin{array}{c}\text { Threat } \\
\text { category }^{5}\end{array}$ \\
\hline & $\begin{array}{l}\text { Rhipsalis hileiabaiana (N.P.Taylor \& Barthlott) N. } \\
\text { Korotkova \& Barthlott }\end{array}$ & PL 521 & Epi & CAF & DD \\
\hline & Rhipsalis lindbergiana K.Schum & PL 367 & Epi & $\mathrm{O}$ & \\
\hline & Rhipsalis neves-armondii K.Schum & PL 513 & Epi & En & \\
\hline & Rhipsalis oblonga Loefgr. & ML 1019 & Epi & En & \\
\hline Clusiaceae & Clusia panapanari (Aubl.) Choisy & AA 7205 & Hep & $\mathrm{O}$ & \\
\hline \multirow{4}{*}{$\begin{array}{l}\text { Cyclanthacea- } \\
\text { e }\end{array}$} & Asplundia gardneri (Hook,) Harling & PL 213 & Hep & WD & \\
\hline & Asplundia maximiliani Harling & PL 506 & Hep & En & \\
\hline & Evodianthus funifer (Poit.) Lindm. & ML 1464 & Hep & WD & \\
\hline & Thoracocarpus bissectus (Vell.) Harling & PL 427 & Hep & WD & \\
\hline \multirow[t]{7}{*}{ Gesneriaceae } & Codonanthe cordifolia Chautems & JJ 4892 & Epi & $\mathrm{CAF}$ & \\
\hline & Codonanthe gracilis (Mart.) Hanst. & PL 411 & Epi & En & \\
\hline & Codonanthe uleana Fritsch & AA 7221 & Epi & WD & \\
\hline & Columnea sanguinea (Pers.) Hanst. & RB 622 & Epi & WD & \\
\hline & Nematanthus albus Chautems & JJ 4907 & Epi & En & \\
\hline & Nematanthus corticola Schrad. & JP 973 & Epi & En & \\
\hline & Nematanthus lanceolatus (Poir.) Chautems & AA 5276 & Fac & $\mathrm{CAF}$ & \\
\hline $\begin{array}{l}\text { Lentibulariac- } \\
\text { eae }\end{array}$ & Utricularia jamesoniana Oliv. ** & JJ 4908 & Epi & $\mathrm{O}$ & \\
\hline \multirow{2}{*}{$\begin{array}{l}\text { Marcgraviac- } \\
\text { eae }\end{array}$} & Marcgravia coriacea Vahl & AA 6679 & Hep & WD & \\
\hline & Marcgravia polyantha Delpino & JJ 4697 & Hep & $\mathrm{CAF}$ & \\
\hline \multirow{4}{*}{$\begin{array}{l}\text { Melastomata- } \\
\text { ceae }\end{array}$} & Bertolonia alternifolia Baumgratz et al. & AJ 170 & Epi & En & \\
\hline & Bertolonia bullata Baumgratz et al. & AA 5979 & Epi & En & \\
\hline & Bertolonia ovata DC. & $\begin{array}{c}\text { WT } \\
14581\end{array}$ & Epi & En & \\
\hline & Pleiochiton blepharodes (DC.) Reginato \& Goldenberg & JJ 4921 & Epi & En & \\
\hline \multirow[t]{3}{*}{ Moraceae } & Ficus arpazusa Casar. & $\begin{array}{c}\text { WT } \\
14112\end{array}$ & Hep & WD & \\
\hline & Ficus castellviana Dugand & PF 2811 & Hep & WD & \\
\hline & Ficus hirsuta Schott & FF 1447 & Hep & $\mathrm{O}$ & \\
\hline Onagraceae & Fuchsia regia (Vand. ex Vell.) Munz & AA 5806 & Hep & $\mathrm{CAF}$ & \\
\hline \multirow[t]{19}{*}{ Orchidaceae } & Acianthera auriculata (Lindl.) Pridgeon \& M.W.Chase & PL 412 & Epi & En & \\
\hline & $\begin{array}{c}\text { Acianthera capanemae (Barb.Rodr.) Pridgeon \& } \\
\text { M.W.Chase * }\end{array}$ & PL 361 & Epi & En & \\
\hline & $\begin{array}{c}\text { Acianthera hygrophila (Barb.Rodr.) Pridgeon \& } \\
\text { M.W.Chase * }\end{array}$ & AF 2682 & Epi & Di & \\
\hline & Anathallis rubens (Lind1.) Pridgeon \& M.W.Chase & PL 251 & Fac & WD & \\
\hline & Anathallis sclerophylla (Lindl.) Pridgeon \& M.W.Chase & AA 5809 & Epi & WD & \\
\hline & $\begin{array}{c}\text { Baptistonia gutfreundiana (Chiron \& V.P.Castro) Chiron \& } \\
\text { V.P.Castro }\end{array}$ & PL 376 & Epi & En & \\
\hline & Baptistonia truncata (Pabst) Chiron \& V.P.Castro * & RP 536 & Epi & En & CR \\
\hline & Brachionidium restrepioides Hoehne \& Pabst & JJ 4677 & Epi & En & VU \\
\hline & Bulbophyllum micropetaliforme J.E.Leite & PL 225 & Epi & En & \\
\hline & Bulbophyllum napellii Lindl. & JJ 5383 & Epi & CAF & \\
\hline & Camaridium carinatum (Barb.Rodr.) Hoehne & AF 2666 & Epi & $\mathrm{O}$ & \\
\hline & Camaridium cf. micranthum M.A. Blanco & AF 2688 & Epi & - & \\
\hline & Catasetum cf. hookeri Lindl. & JJ 4385 & Epi & - & \\
\hline & Catasetum purum Nees \& Sinnings & PL 244 & Epi & En & \\
\hline & Cirrhaea cf. seidelii Pabst & PL 355 & Epi & - & \\
\hline & Coppensia flexuosa (Sims) Campacci & JJ 4922 & Epi & $\mathrm{Di}$ & \\
\hline & Coppensia hookeri (Rolfe) F.Barros \& L.Guimarães & AA 6626 & Epi & En & \\
\hline & Dichaea brevicaulis Cogn. & PL 354 & Epi & En & \\
\hline & Dichaea cogniauxiana Schltr. & AA 5816 & Epi & $\mathrm{CAF}$ & \\
\hline
\end{tabular}


Table 1. Continued.

\begin{tabular}{|c|c|c|c|c|c|}
\hline Family & Specie $^{1}$ & Voucher $^{2}$ & Habit $^{3}$ & Distribution $^{4}$ & $\begin{array}{c}\text { Threat } \\
\text { category }\end{array}$ \\
\hline & Dichaea pendula (Aubl.) Cogn. & PL 353 & Epi & WD & \\
\hline & Elleanthus brasiliensis (Lindl.) Rchb.f. & PL 549 & Epi & $\mathrm{O}$ & \\
\hline & Elleanthus crinipes Rchb.f. & AF 2673 & Epi & $\mathrm{CAF}$ & \\
\hline & Elleanthus hymenophorus (Rchb.f.) Rchb.f. *** & AA 4960 & Epi & WD & \\
\hline & Elleanthus linifolius C.Presl & AA 4983 & Epi & WD & \\
\hline & Encyclia fimbriata A.C.Bastos et al. & $\begin{array}{c}\text { WT } \\
14604\end{array}$ & Epi & En & \\
\hline & Encyclia patens Hook. & PL 326 & Fac & CAF & \\
\hline & Encyclia unaensis Fowlie & AF 2659 & Epi & En & \\
\hline & Epidendrum armeniacum Lindl. & AA 6581 & Epi & $\mathrm{O}$ & \\
\hline & Epidendrum densiflorum Lindl. & PL 463 & Fac & WD & \\
\hline & Epidendrum latilabre Lindl. * & PL 432 & Epi & Di & \\
\hline & Epidendrum macrocarpum Rich. & AA 6413 & Epi & $\mathrm{O}$ & \\
\hline & Epidendrum nocturnum Jacq. & ML 539 & Epi & WD & \\
\hline & Epidendrum ochrochlorum Barb.Rodr. & JP 971 & Epi & WD & \\
\hline & Epidendrum paranaense Barb.Rodr. & AA 6412 & Fac & WD & \\
\hline & Epidendrum ramosum Jacq. & AA 5810 & Epi & CAF & \\
\hline & Epidendrum saximontanum Pabst & AF 2685 & Epi & En & \\
\hline & Epidendrum secundum Jacq. & JJ 4901 & Epi & WD & \\
\hline & Epidendrum $\mathrm{sp}$ & JJ 4904 & Epi & - & \\
\hline & Gomesa recurva Barb.Rodr. & AA 4448 & Fac & $\mathrm{CAF}$ & \\
\hline & Grandiphyllum pohlianum (Cogn.) Docha Neto * & PL 413 & Epi & En & \\
\hline & Heterotaxis brasiliensis (Brieger \& Illg) F.Barros & PL 191 & Epi & $\mathrm{Di}$ & \\
\hline & Houlletia brocklehurstiana Lindl. & $\begin{array}{c}\text { WT } \\
14594\end{array}$ & Epi & En & EN \\
\hline & Jacquiniella globosa (Jacq.) Schltr. & AF 2675 & Epi & WD & \\
\hline & Koellensteinia graminea Rchb.f. & PL 555 & Epi & $\mathrm{O}$ & \\
\hline & Lankesterella longicollis (Cogn.) Hoehne * & AF 2684 & Epi & En & \\
\hline & Lockhartia lunifera Rchb.f. & AF 2654 & Epi & Di & \\
\hline & Masdevallia infracta Lindl. & $\begin{array}{c}\text { WT } \\
14097\end{array}$ & Epi & CAF & \\
\hline & Maxillaria bradei Schltr. ex Hoehne * & JJ 4912 & Epi & En & \\
\hline & Maxilaria candida Lodd. ex Lindl. * & ML 1023 & Epi & Di & \\
\hline & Maxillaria leucaimata Barb.Rodr. & AA 5329 & Epi & Di & \\
\hline & Maxillaria ochroleuca Lodd. ex Lindl. & JJ 4379 & Epi & WD & \\
\hline & Maxilaria aff. ringens Rchb.f. & PL 154 & Epi & - & \\
\hline & Maxillaria spiritusanctensis Pabst & JJ 4681 & Epi & En & \\
\hline & Maxilaria aff. splendens Poepp. \& Endl. & PL 374 & Epi & - & \\
\hline & Maxilaria $\mathrm{sp}$ & PL 314 & Epi & - & \\
\hline & $\begin{array}{c}\text { Maxillariella robusta (Barb. Rodr.) M.A.Blanco \& } \\
\text { Carnevali * }\end{array}$ & PL 316 & Epi & En & \\
\hline & Mormolyca rufescens (Lindl.) M.A.Blanco & JJ 4683 & Epi & Di & \\
\hline & Nitidobulbon nasutum (Rchb.f.) Ojeda \& Carnevali & AA 4970 & Epi & WD & \\
\hline & Octomeria concolor Barb.Rodr. * & PL 464 & Epi & $\mathrm{O}$ & \\
\hline & Octomeria crassifolia Lindl. & AF 2657 & Epi & $\mathrm{O}$ & \\
\hline & Octomeria decumbens Cogn. & PL 400 & Epi & En & DD \\
\hline & Octomeria geraensis Barb.Rodr. * & PL 265 & Epi & En & VU \\
\hline & Octomeria grandiflora Lindl. & PL 226 & Epi & WD & \\
\hline & Octomeria tricolor Rchb.f. & JJ 4665 & Epi & En & \\
\hline & Octomeria $\mathrm{sp}$ & PL 493 & Epi & - & \\
\hline & Ornithidium rigidum (Barb.Rodr.) M.A.Blanco \& Ojeda * & RP 301 & Epi & Di & \\
\hline & Pabstiella bradei (Schltr.) Luer * & PL 377 & Epi & En & \\
\hline & Pabstiella ramphastorhyncha (Barb. Rodr.) L. Kollmann & PL 360 & Epi & CAF & \\
\hline & Pabstiella aff. trifida (Lind1.) Luer & AF 2658 & Epi & - & \\
\hline & Pleurothallis ruscifolia (Jacq.) R.Br. & PF 2821 & Epi & WD & \\
\hline & Pleurothallis sp. & PL 222 & Epi & - & \\
\hline
\end{tabular}


Table 1. Continued.

\begin{tabular}{|c|c|c|c|c|c|}
\hline Family & Specie $^{1}$ & Voucher $^{2}$ & Habit $^{3}$ & Distribution $^{4}$ & $\begin{array}{c}\text { Threat } \\
\text { category }^{5}\end{array}$ \\
\hline & Polycycnis silvana F.Barros & AA 5805 & Epi & En & \\
\hline & Polystachya estrellensis Rchb.f. & RP 294 & Fac & WD & \\
\hline & Promenaea silvana F.Barros \& Cath. & JJ 4815 & Epi & En & \\
\hline & Promenaea xanthina Lindl. & RP 330 & Epi & En & \\
\hline & Prosthechea bueraremensis (Campacci) Campacci & AF 2662 & Epi & En & \\
\hline & Prosthechea calamaria (Lindl.) W.E.Higgins * & AF 2674 & Epi & En & \\
\hline & Prosthechea fragans (Sw.) W.E.Higgins & AA 5361 & Epi & WD & \\
\hline & Prosthechea pachysepala (Klotzsch) Chiron \& V.P.Castro & RP 335 & Epi & $\mathrm{CAF}$ & \\
\hline & Prosthechea pygmaea (Hook.) W.E.Higgins & $\begin{array}{c}\text { WT } \\
14101\end{array}$ & Epi & WD & \\
\hline & Prosthechea vespa (Vell.) W.E.Higgins * & ML 549 & Fac & WD & \\
\hline & Rhetinantha notylioglossa (Rchb.f.) M.A.Blanco & AA 5355 & Epi & Di & \\
\hline & Scaphyglottis fusiformis (Griseb.) Schultes * & PL 399 & Epi & WD & \\
\hline & Scaphyglottis modesta (Rchb.f.) Schltr. & AF 2672 & Fac & WD & \\
\hline & Scaphyglottis reflexa Lindl. & $\begin{array}{c}\text { WT } \\
14096\end{array}$ & Epi & $\mathrm{O}$ & \\
\hline & Sobralia cf. sessilis Lindl. & AF 2651 & Epi & - & \\
\hline & Specklinia grobyi Batem. ex Lindl. & PL 494 & Epi & WD & \\
\hline & Stelis aprica Lindl. & AA 6588 & Epi & WD & \\
\hline & Stelis caespitosa Lindl. ** & PL 532 & Epi & $\mathrm{O}$ & \\
\hline & Stelis deregularis Barb.Rodr. & $\begin{array}{c}\text { WT } \\
14102\end{array}$ & Epi & En & \\
\hline & Stelis aff. fraterna Lindl. & PL 256 & Epi & - & \\
\hline & Stelis intermedia Poepp. \& Endl. * & AA 5746 & Epi & $\mathrm{O}$ & \\
\hline & Stelis megantha Barb.Rodr. * & PL 135 & Epi & En & \\
\hline & Stelis papaquerensis Rchb.f. * & PL 430 & Epi & $\mathrm{Di}$ & \\
\hline & Stelis pauciflora Lindl. * & PL 175 & Epi & $\mathrm{Di}$ & \\
\hline & Stelis ruprechtiana Rchb.f. * & PL 359 & Epi & En & \\
\hline & Stelis sp. 1 & PL 431 & Epi & - & \\
\hline & Stelis sp. 2 & PL 519 & Epi & - & \\
\hline & Stelis sp. 3 & JJ 4713 & Epi & - & \\
\hline & Stelis sp. 4 & $\begin{array}{c}\text { WT } \\
14103\end{array}$ & Epi & - & \\
\hline & Stelis sp. 5 & ML 1187 & Epi & - & \\
\hline & Trichopilia santoslimae Brade * & PL 221 & Epi & En & CR \\
\hline & Xylobium colleyi (Bateman ex Lind1.) Rolfe * & RP 69 & Epi & WD & \\
\hline & Xylobium variegatum (Ruiz \& Pav.) Mansf. * & PL 310 & $\mathrm{Fac}$ & WD & \\
\hline & Zygopetalum crinitum Lodd. * & AA 7264 & Epi & En & \\
\hline & Zygopetalum cf. maxillare Lodd. & AF 2665 & Epi & - & \\
\hline \multirow[t]{14}{*}{ Piperaceae } & Peperomia alata Ruiz \& Pav. & JJ 4346 & Fac & WD & \\
\hline & Peperomia corcovadensis Gardner * & PL 100 & Fac & $\mathrm{O}$ & \\
\hline & Peperomia elongata Kunth & AA 6649 & $\mathrm{Fac}$ & WD & \\
\hline & Peperomia emarginella (Sw.) C.DC. & $\begin{array}{c}\text { WT } \\
14541\end{array}$ & $\mathrm{Fac}$ & $\mathrm{O}$ & \\
\hline & Peperomia hernandiifolia (Vahl) A.Dietr. & ML 1020 & Fac & WD & \\
\hline & Peperomia macrostachya (Vahl) A.Dietr. & JJ 4798 & Epi & WD & \\
\hline & Peperomia magnoliifolia (Jacq.) A.Dietr. & AA 5952 & Fac & WD & \\
\hline & Peperomia obtusifolia (L.) A.Dietr. & AA 5946 & Fac & WD & \\
\hline & Peperomia pilicaulis C.DC. & MJ 840 & Fac & WD & \\
\hline & Peperomia pseudoestrelensis C.DC. & AA 6357 & Fac & En & \\
\hline & Peperomia serpens (Sw.) Loud & PL 548 & Fac & WD & \\
\hline & Peperomia tenella (Sw.) A.Dietr. & PL 421 & Fac & CAF & \\
\hline & Peperomia tetraphylla (G.Forst.) Hook. \& Arn. & AA 6681 & Fac & WD & \\
\hline & Peperomia urocarpa Fisch. \& C.A.Mey. & AA 5287 & $\mathrm{Fac}$ & WD & \\
\hline \multirow[t]{3}{*}{ Rubiaceae } & Hillia parasitica Jacq. & AA 5800 & Epi & WD & \\
\hline & Notopleura bahiensis C.M.Taylor & AA 5741 & Epi & En & \\
\hline & Schradera polycephala DC. & JJ 4851 & Hep & $\mathrm{Di}$ & \\
\hline Solanaceae & Dyssochroma viridiflorum (Sims) Miers & AA 4962 & Hep & En & \\
\hline
\end{tabular}


Epiphytic angiosperms in a mountain forest in southern Bahia, Brazil

Table 2. Comparison of species richness for the main families and genera with epiphytic habit between Serra das Lontras National Park and other surveys carried at the Atlantic Forest. ${ }^{1}$ N.P. - National Park; S.P. - State Park; N.R. - Natural Reserve. "-" - Information not available.

\begin{tabular}{|c|c|c|c|c|c|c|c|c|}
\hline Study area ${ }^{1}$ & $\begin{array}{l}\text { Serra das } \\
\text { Lontras } \\
\text { N.P., BA }\end{array}$ & $\begin{array}{l}\text { Macaé de } \\
\text { Cima, RJ }\end{array}$ & $\begin{array}{l}\text { Upper } \\
\text { Iguaçu } \\
\text { River } \\
\text { Basin, PR }\end{array}$ & $\begin{array}{l}\text { Prata } \\
\text { Moutain } \\
\text { Range, } \\
\text { PR }\end{array}$ & $\begin{array}{l}\text { Ibitipoca } \\
\text { S.P., MG }\end{array}$ & $\begin{array}{l}\text { Carlos } \\
\text { Botelho } \\
\text { S.P., SP }\end{array}$ & $\begin{array}{l}\text { Ilha do } \\
\text { Cardoso } \\
\text { S.P., SP }\end{array}$ & $\begin{array}{l}\text { Vale do Rio } \\
\text { Doce N.R., } \\
\text { ES }\end{array}$ \\
\hline Taxon & $\begin{array}{l}\text { Present } \\
\text { study }\end{array}$ & $\begin{array}{l}\text { Fontoura } \\
\text { et al. } 1997 \text {; } \\
\text { Costa \& } \\
\text { Wendt } \\
2007\end{array}$ & $\begin{array}{l}\text { Kersten \& } \\
\text { Kunyioshi } \\
2006\end{array}$ & $\begin{array}{l}\text { Blum } \\
\text { et al. } 2011\end{array}$ & $\begin{array}{l}\text { Menini } \\
\text { Neto et al. } \\
2009\end{array}$ & $\begin{array}{l}\text { Breier } \\
\text { et al. } 2005\end{array}$ & $\begin{array}{l}\text { Breier } \\
\text { et al. } 2005\end{array}$ & $\begin{array}{l}\text { Coelho } \\
2010\end{array}$ \\
\hline Total area & $11,000 \mathrm{ha}$ & 7,000ha & - & 6.3ha & $1,923.5 \mathrm{ha}$ & $10.24 \mathrm{ha}$ & $10.24 \mathrm{ha}$ & $22.000 \mathrm{ha}$ \\
\hline $\begin{array}{l}\text { Total epiphytic } \\
\text { Angiosperms }\end{array}$ & 256 & 275 & 254 & 204 & 181 & 121 & 136 & - \\
\hline Orchidaceae & 103 & 184 & 147 & 103 & 66 & 42 & 72 & - \\
\hline Bromeliaceae & 62 & 45 & 39 & 38 & 22 & 27 & 33 & - \\
\hline Araceae & 29 & 5 & 9 & 14 & 9 & 13 & 11 & 22 \\
\hline Piperaceae & 15 & 5 & 24 & 14 & 3 & 8 & 6 & - \\
\hline Cactaceae & 7 & 7 & 12 & 9 & 7 & 11 & 1 & - \\
\hline Gesneriaceae & 7 & 6 & 3 & 5 & 2 & 6 & 3 & - \\
\hline Vriesea & 22 & 18 & 11 & 18 & 7 & 12 & 15 & - \\
\hline Peperomia & 15 & 5 & 22 & 14 & 3 & 7 & 5 & - \\
\hline Stelis & 14 & 4 & 6 & 7 & 5 & 1 & 1 & - \\
\hline Anthurium & 13 & - & 2 & 6 & 5 & 3 & 3 & 4 \\
\hline Philodendron & 12 & 10 & 7 & 6 & 4 & 8 & 7 & 12 \\
\hline Aechmea & 11 & 4 & 9 & 5 & 1 & 5 & 3 & - \\
\hline Epidendrum & 11 & 10 & 4 & 12 & 6 & 4 & 8 & - \\
\hline Hohenbergia & 9 & 0 & 0 & 0 & 0 & 0 & 0 & - \\
\hline
\end{tabular}

Aechmea, and Hohenbergia also exhibited their greatest epiphytic species diversity in the SLNP. It is interesting that Hohenbergia was among the richest genera in the study area, as this genus does not commonly figure among the most species rich taxa in epiphyte surveys. The greatest diversity of this genus is encountered in southern Bahia, where 21 of its 25 Atlantic Forest species occur (Martinelli et al. 2008, Forzza et al. 2013).

In addition to $H$. brachycephala and $H$. minor, both endemic to Bahia, H. edmundoi, a poorly-known species from Bahia for which no habitat information was available (Smith \& Downs 1979, Forzza et al. 2013), was found in a submontane forest in the SLNP; H. augusta, previously considered to be restricted to southeastern Brazil, was also collected. These results reaffirm the importance of southern Bahia State in the distribution and taxonomy of this genus.

Vriesea (Tillandsioideae) and Aechmea (Bromelioideae) represent $12 \%$ and $7 \%$, respectively, of the total number of species in the Atlantic Forest (Forzza et al. 2013). Fontoura et al. (2012) reported that Bromelioideae taxa were encountered 1.6 times more frequently in local surveys of epiphytic species in the Atlantic Forest than representatives of Tillandsioideae. This proportion was lower in the SLNP, where Vriesea is very diverse, with almost the same numbers of species being encountered in each subfamily (34 spp. Bromelioideae x 30 spp. Tillandsioideae).

Another family that demonstrated elevated richness in the SLNP was Araceae, with one third of the 65 species known to the Atlantic Forest being encountered there (Coelho et al. 2013). The most diversified genera of this family were likewise well represented in the park, with more than half of the species of Anthurium and Philodendron known from Bahia being encountered in the SLNP. Additionally, two new occurrence records for Bahia State (A. boudetii and $P$. edmundoi), one for the Atlantic Forest domain (A. gracile), and five morphotypes of Anthurium reinforce the importance of additional studies in mountainous areas there to improve our knowledge of the distribution of this family within the state. In contrast to the high endemism levels of Bromeliaceae and Orchidaceae, representatives of Araceae exhibit wide (and sometimes disjunct) distributions (Coelho et al. 2013).

Orchidaceae is responsible for approximately $70 \%$ of the global epiphytic flora, and is always among the top families in every floristic study of that habit (Benzing 1990, Kersten 2010, Zotz 2013). Most orchid diversity is found in the tropics, especially in mountainous areas (Dressler 1993). The majority of the orchid taxa encountered in the SLNP is relatively small (e.g., Acianthera, Anathallis, Brachionidium, Jacquiniella, Lankesterella, Octomeria, Pabstiella, Pleurothallis, Promenaea, Specklinia, and Stelis) and commonly neglected in floristic surveys - and therefore poorly represented in scientific collections (Dressler 2005). As the present study focused on epiphytes (and ex-situ cultivation was undertaken), high numbers of species were included on the final list - with 22 new occurrences for Bahia State (Table 1).

Northeastern Brazil represents one of the 10 areas in the world considered most likely to have undescribed species, and where models predict a 30\% increase in known diversity (Joppa et al. 2011). Since initiating preliminary studies of the flora of the SLNP (Amorim et al. 2009), six new epiphyte species have 
been described for the area: Bertolonia alternifolia, B. bullata, Clistax bahiensis, Encyclia fimbriata, Quesnelia clavata, and Vriesea longisepala, and one is about to be published. The present survey found other possible taxonomic novelties, and recent studies in neighboring areas encountered several new species with epiphytic habits (e.g., Amorim \& Leme 2009, Leme \& Kollmann 2011, Costa et al. 2012, Reginato et al. 2013) emphasizing the need of further investigations of this group in southern Bahia.

The present study expands the distribution of many taxa, with one new occurrence for Brazil (Elleanthus hymenophorus (Rchb.f.) Rchb.f.), four for the Atlantic Forest biome, and 30 for Bahia State (Table 1). Utricularia jamsoniana Oliv., a species previously known only from altitudinal regions of Central America and northern South America (Taylor 1989), was encountered in a cloud forest in the highest area of the park (Peito de Moça and Javi mountain ranges). Over half (16 spp.) of these new records were known only from collections in strictly southern and southeastern regions. The epiphytic flora of the SLNP shares more species with southern and southeastern Brazil (71 spp.) than with northeastern states (five species - four Aechmea and one Hohenbergia). This same pattern was found for ferns and lycophytes on a nearby mountainous area in the Serra Bonita Private Reserve, with almost a quarter of the surveyed species being previously restricted to southeastern Brazil (Matos et al. 2010).

Almost half of the 221 taxa identified to species level in the present study are endemic to the Atlantic Forest (100 spp. - 45\%) (Table 1), and most of them belong to Bromeliaceae and Orchidaceae (44 and 32 species respectively). These two families exhibit high degrees of endemism in the Atlantic Forest, with $80 \%$ of Bromeliaceae and $63 \%$ of Orchidaceae being restricted to this domain (Martinelli et al. 2008, Stehmann et al. 2009). These high levels of endemism may be related to limited connections with the other major South American forest formation in the Amazon (Mori et al. 1981). These two forests are separated by an open area known as the "dry diagonal" (Prado \& Gibbs 1993) that acts as a barrier to species dispersion, especially less droughttolerant taxa.

On the other hand, 60 species showed broad distributions in Brazil or the Neotropical region, and 24 had disjunct distributions between the Atlantic and Amazon forests (Table 1); 19 species also occur in the "Cerrado" (Brazilian savanna) region, suggesting that dry formations do not act as barriers to all epiphyte taxa, especially those with broader ecophysiological tolerances (Oliveira-Filho \& Ratter 1995, Ibisch et al. 1996). Deciduous and semi-deciduous seasonal forest patches and gallery forests that crisscross the Cerrado may offer dispersal pathways for many species and establish tenuous connections between these two major forest formations (Leitão Filho 1987, Oliveira-Filho \& Ratter 1995).

All of the 10 species cited on the Red Book of Brazilian Flora (Martinelli \& Moraes 2013) as threatened belong to Bromeliaceae and Orchidaceae, two very ornamental families. The five bromeliads are restricted to Northeast Brazil, and four of them are endemic to Bahia. On the other hand, the orchids indicated as threatened are new occurrences to the state. These results reinforce the importance of the park as a conservation unit and the relevance of floristic surveys, especially for often neglected groups such as epiphytes. Recent floristic studies in mountainous areas in southern Bahia have revealed large numbers of new species and new occurrences of epiphytes pointing to the need for further investigations of this group.

\section{Acknowledgments}

We thank FAPESB, FAPERJ and CAPES for fieldwork financial support. Paula Leitman thank CNPq and CAPES for master's scholarship. Rafaela C. Forzza and André Amorim are $\mathrm{CNPq}$ research fellows. We also thank the specialists who helped identifying the species - Alain Chautems, Andrea Costa, Charlotte Taylor, Claudio Nicoletti Fraga, Daniela Zappi, Daniele Monteiro, Eliane de Lima Jacques, Elton Leme, Elsie Franklin Guimarães, João Renato Stehmann, Ludovic Kollmann, Marcus Nadruz, Nigel Taylor, Ricardo Perdiz, Sheila Profice \& Simon Mayo - and fieldwork helpers - José Lima Paixão, Carlinhos, Lukas Daneu and Ricardo Perdiz.

\section{References}

AMORIM, A.M.A. \& LEME, E.M.C. 2009. Two new species of Quesnelia (Bromeliaceae: Bromelioideae) from the Atlantic Rainforest of Bahia, Brazil. Brittonia 61(1): 14-21. doi: http:// dx.doi.org/10.1007/s12228-008-9049-5

AMORIM, A.M.A., JARDIM, J.G., LOPES, M.M.M., FIASCHI, P., BORGES, R.A.X., PERDIZ, R.O. \& THOMAS, W.W. 2009. Angiospermas em remanescentes de Floresta Montana no sul da Bahia, Brasil. Biota Neotrop. 9(3): 313-348 http://www.biotaneotropica.org.br/v9n3/en/abstract?inventory+bn02909032009 (accessed in 15/07/2013). doi: http://dx.doi.org/10.1590/S167606032009000300028

BENZING, D.H. 1990. Vascular epiphytes. Cambridge University Press, New York.

BLUM, C.T., RODERJAN, C.V. \& GALVÃO, F. 2011. Composição florística e distribuição altitudinal de epífitas vasculares da Floresta Ombrófila Densa na Serra da Prata, Morretes, Paraná, Brasil. Biota Neotrop. 11(4): 1-19 http://www.biotaneotropica.org.br/ v11n4/en/abstract?inventory+bn00811042011 (accessed in 15/07/ 2013). doi: http://dx.doi.org/10.1590/S1676-06032011000400015

BORGO, M. \& SILVA, S.M. 2003. Epífitos vasculares em fragmentos de Floresta Ombrófila Mista, Curitiba, Paraná, Brasil. Rev. Bras. Bot. 26(3): 391-401. doi: http://dx.doi.org/10.1590/S010084042003000300012

BREIER T.B. 2005. O epifitismo vascular em florestas do Sudeste do Brasil. PhD Thesis, Universidade Estadual de Campinas, Campinas.

BUZATTO, C.R., SEVERO, B.M.A. \& WAECHTER, J.L. 2008. Composição florística e distribuição ecológica de epífitos vasculares na Floresta Nacional de Passo Fundo, Rio Grande do Sul. Iheringia, Bot. 63(2): 231-239.

COELHO, M.A.N. 2010. A família Araceae na Reserva Natural Vale, Linhares, Espírito Santo, Brasil. Bol. Mus. Biol. Mello Leitão 28: 41-87.

COELHO, M.A.N., SAKURAGUI, C.M., MAYO, S., SOARES, M.L., TEMPONI, L.G., CALAZANS, L.S.B., GONçALVES, E.G., \& ANDRADE, I.M. 2013. Araceae. In Lista de Espécies da Flora do Brasil. Jardim Botânico do Rio de Janeiro. http:// floradobrasil.jbrj.gov.br/jabot/floradobrasil/FB51 (accessed in 15/ 05/2013).

COSTA, A. \& WENDT, T. 2007. Bromeliaceae na região de Macaé de Cima, Nova Friburgo, Rio de Janeiro, Brasil. Rodriguésia 58(4): 905-939.

COSTA, A.F., FOnTOURA, T. \& AMORIM, A.M. 2012. Novelties in Bromeliaceae from the northeastern Brazilian Atlantic Rainforest. J. Torrey Bot. Soc. 139(1): 34-45. doi: http:// dx.doi.org/10.3159/TORREY-D-11-00061.1

DRESSLER, R.L. 1993. Phylogeny and classification of the orchid family. Dioscorides Press, Portland. 
DRESSLER, R.L. 2005. How many orchid species? Selbyana 26(1,2): 155-158.

FONTOURA, T., SYLVESTRE, L.S., VAZ, A.M.S.F. \& VIEIRA, C.M. 1997. Epífitas vasculares, hemiepífitas e hemiparasitas da Reserva Ecológica de Macaé de Cima. In Serra de Macaé de Cima: Diversidade florística e conservação em Mata Atlântica (H.C. LIMA \& R.R. GUEDES-BRUNI, EDS.). JARDIM BOTÂNICO DO RIO DE JANEIRO, RIO DE JANEIRO, P. 89-101.

FONTOURA, T., SCUDELlER, V.V. \& COSTA, A.F. 2012. Floristics and environmental factors determining the geographic distribution of epiphytic bromeliads in the Brazilian Atlantic Rain Forest. Flora (Jena), 9(): 662-672. doi: http://dx.doi.org/10.1016/ j.flora.2012.05.003

FORZZA, R.C., COSTA, A., SIQUEIRA FILHO, J.A., MARTINELLI, G., MONTEIRO, R.F., SANTOS-SILVA, F., SARAIVA, D. P. \& PAIXÃO-SOUZA, B. 2013. Bromeliaceae. In Lista de Espécies da Flora do Brasil. Jardim Botânico do Rio de Janeiro. http://floradobrasil.jbrj.gov.br/jabot/floradobrasil/FB66 (accessed in 15/05/2013).

GENTRY, A.H. \& DODSON, C.H. 1987. Diversity and biogeography of neotropical vascular epiphytes. Ann. Missouri Bot. Gard. 74: 205-233. doi: http://dx.doi.org/10.2307/2399395

GIONGO, C. \& WAECHTER, J.L. 2004. Composição florística e estrutura comunitária de epífitos vasculares em uma floresta de galeria na Depressão Central do Rio Grande do Sul. Rev. Bras. Bot. 27(3): 563-572. doi: http://dx.doi.org/10.1590/S010084042004000300015

IBISCH, P.L., BOEGNER, A., NIEDER, J. \& BARTHLOTT, W. 1996. How diverse are neotropical epiphytes? An analysis based on the "Catalogue of the flowering plants and gymnosperms of Peru". Ecotropica 2: 13-28.

JOPPA, L.N., ROBERTS, D.L., MYERS, N. \& PIMM, S.L. 2011. Biodiversity hotspots house most undiscovered plant species. P. Natl. Acad. Sci. USA 108(32): 13171-13176. doi: http://dx.doi.org/ 10.1073/pnas. 1109389108

KERSTEN, R.A. 2010. Epífitas vasculares: histórico, participação taxonômica e aspectos relevantes, com ênfase na Mata Atlântica. Hoehnea 37(1): 9-38. doi: http://dx.doi.org/10.1590/S223689062010000100001

KERSTEN, R.A. \& SILVA, S.M. 2001. Composição florística e estrutura do componente epifítico vascular em floresta da planície litorânea na Ilha do Mel, Paraná, Brasil. Rev. Bras. Bot. 24(2): 213-226. doi: http://dx.doi.org/10.1590/S0100-84042001000200012

KÜPER, W., KREFT, H., NIEDER, J., KÖSTER, N. \& BARTHLOTT, W. 2004. Large-scale diversity patterns of vascular epiphytes in Neotropical montane rain forests. J. biogeogr. 31: 1477-1487. doi: http://dx.doi.org/10.1111/j.1365-2699.2004.01093.x

LEITÃO FILHO, H.F. 1987. Considerações sobre a florística de florestas tropicais e subtropicais do Brasil. IPEF 35: 41-46.

LEME, E.M.C. \& KOLLMANN, L.J.C. 2011. New species and a new combination of Brazilian Bromeliaceae. Phytotaxa 16: 1-36.

MADISON, M. 1977. Vascular epiphytes: their systematic occurrence and salient features. Selbyana 2: 1-13.

MANIA, L. \& MONTEIRO, R. 2010. Florística e ecologia de epífitas vasculares em um fragmento de floresta de restinga, Ubatuba, SP, Brasil. Rodriguésia 61(4): 705-713.

MARTINELLI, G., VIEIRA, C.M., GONZALEZ, M., LEITMAN, P., PIRATININGA, A., COSTA, A.F. \& FORZZA, R.C. 2008. Bromeliaceae da Mata Atlântica Brasileira: lista de espécies, distribuição e conservação. Rodriguésia 59(1): 209-258.

MARTINELLI, G. \& MORAES, M.A. (orgs.) 2013. Livro vermelho da flora do Brasil. Andrea Jakobsson/Instituto de Pesquisas Jardim Botânico do Rio de Janeiro, Rio de Janeiro. 1100 p.

MARTINI, A.M.Z., FIASCHI, P., AMORIM, A.M. \& PAIXÃO, J.L. 2007. A hot-point within a hot-spot: a high diversity site in Brazil's Atlantic Forest. Biodivers. Conserv. 16(11): 3111-3128. doi: http:// dx.doi.org/10.1007/s10531-007-9166-6
MARTINS, S.E., ROSSI, L., SAMPAIO, P.S.P. \& MAGENTA, M.A.G. 2008. Caracterização florística de comunidades vegetais de restinga em Bertioga, SP, Brasil. Acta bot. bras. 22(1): 249-274. doi: http://dx.doi.org/10.1590/S0102-33062008000100024

MATOS, F.B., AMORIM, A.M. \& LABIAK, P.H. 2010. The ferns and lycophytes of a montane tropical forest in southern Bahia, Brazil. J. Bot. Res. Inst. Texas 4(1): 333-346.

MENINI NETO, L., FORZZA, R.C., \& ZAPPI, D. 2009. Angiosperm epiphytes as conservation indicators in forest fragments: a case study from southeastern Minas Gerais, Brazil. Biodivers. Conserv. 18: 3785-3807. doi: http://dx.doi.org/10.1007/s10531-009-9679-2

MORI, S., BOOM, B.M. \& PRANCE, G.T. 1981. Distribution patterns and conservation of eastern Brazilian coastal forest tree species. Brittonia 33(2): 233-245. doi: http://dx.doi.org/10.2307/ 2806330

MORI, S., BOOM, B.M., CARVALHO, A.M. \& SANTOS, T.S. 1983. Southern Bahian moist forest. Bot. rev. 49(2): 155-232. doi: http:// dx.doi.org/10.1007/BF02861011

NACIF, P.G.S., COSTA, O.V., ARAÚJO, M. \& SANTOS, P.S. 2009. Geomorfodinâmica da Região do Complexo de Serras das Lontras. In Complexo de Serras das Lontras e Una, Bahia: Elementos naturais e aspectos de sua conservação (Save Brasil, IESB e Birdlife International, eds.). SAVE Brasil, São Paulo. p. 9-14.

NIEDER, J., PROSPERí, J. \& MICHALOUD, G. 2001. Epiphytes and their contribution to canopy diversity. Plant ecol. 153: 51-63. doi: http://dx.doi.org/10.1023/A:1017517119305

OLIVEIRA-FILHO, A.T. \& RATTER, J.A. 1995. A study of the origin of central Brazilian forests by the analysis of plant species distribution patterns. Edinb. j. bot. 52(2): 141-194. doi: http:// dx.doi.org/10.1017/S0960428600000949

OLIVEIRA-FILHO, A.T. \& FONTES, M.A.L. 2000. Patterns of floristic differentiation among Atlantic forests in southeastern Brazil and the influence of climate. Biotropica 32(4b): 793-810. doi: http://dx.doi.org/10.1111/j.1744-7429.2000.tb00619.x

PEEL, M.C., FINLAYSON, B.L. \& MCMAHON, T.A. 2007. Updated world map of the Köppen-Geiger climate classification. Hydrol. Earth Syst. Sc. 11: 1633-1644. doi: http://dx.doi.org/ 10.5194/hess-11-1633-2007

PRADO, D. \& GIBBS, P. 1993. Patterns of species distributions in the dry seasonal forests of South America. Ann. Missouri Bot. Gard. 80(4): 902-927. doi: http://dx.doi.org/10.2307/2399937

REGINATO, M., BAUMGRATZ, J.F.A. \& GOLDENBERG, R. 2013. A taxonomic revision of Pleiochiton (Melastomataceae, Miconieae). Brittonia 65(1): 16-41. doi: http://dx.doi.org/10.1007/ s12228-012-9258-9

ROCHA, D.S.B. \& AMORIM, A.M.A. 2012. Heterogeneidade altitudinal na Floresta Atlântica setentrional: um estudo de caso no sul da Bahia, Brasil. Acta bot. bras. 26: 309-327.

SAVE Brasil, IESB \& BirdLife International 2009. Complexo Serra das Lontras e Una, Bahia: Elementos naturais e aspectos de sua conservação. SAVE Brasil, São Paulo.

SMITH, L.B. \& DOWNS, R.J. 1979. Bromelioideae (Bromeliaceae). Flora neotrop. 14(3): 1-649.

STADTMÜLLER, T. 1987. Cloud Forests in the Humid Tropics: A bibliographic review. http://archive.unu.edu/unupress/unupbooks/ 80670e/80670E00.htm\#Contents (accessed in 08/2012).

STEHMANN, J.R., FORZZA, R.C., SOBRAL, M. \& KAMINO, L.H.Y. 2009. Gimnospermas e Angiospermas. In Plantas da Floresta Atlântica (J.R. STEHMANN, R.C. FORZZA, A. SALINO, M. SOBRAL, D.P. COSTA \& L.H.Y. KAMINO, EDS.). JARDIM BOTÂNICO DO RIO DE JANEIRO, RIO DE JANEIRO. P. 27-37.

SUDGEN, A. \& ROBINS, R. 1979. Aspects of the Ecology of Vascular Epiphytes in Colombian Cloud Forests, I. The Distribution of the epiphytic flora. Biotropica 11(3): 173-188. doi: http://dx.doi.org/ $10.2307 / 2388037$

TAYLOR, P. 1989. The genus Utricularia - a taxonomic monograph. Kew Bulletin, Additional Series XIV, London. 
THOMAS, W.W., CARVAlho, A.M.V., AMORIM, A.M., GARRISON, J. \& ARBELÁEZ, A.L. 1998. Plant endemism in two forests in southern Bahia, Brazil. Biodivers. Conserv. 7(3): 311322. doi: http://dx.doi.org/10.1023/A:1008825627656

THOMAS, W.W., JARDIM, J.G., FIASCHI, P., MARIANO-NETO, E. \& AMORIM, A.M. 2009. Composição florística e estrutura do componente arbóreo de uma área transicional de Floresta Atlântica no sul da Bahia, Brasil. Rev. Bras. Bot. 32(1): 65-78. doi: http://
dx.doi.org/10.1590/S0100-84042009000100007

WOLF, J.H.D. \& FLAMENCO-S, A. 2003. Patterns in species richness and distribution of vascular epiphytes in Chiapas, Mexico. J. biogeogr. 30: 1689-1707. doi: http://dx.doi.org/10.1046/j.13652699.2003.00902.x

ZOTZ, G. 2013. The systematic distribution of vascular epiphytes - a critical update. Bot. j. Linn. Soc. 171: 453-481. doi: http:// dx.doi.org/10.1111/boj.12010 